\title{
Reliability of High Power Laser Diode Arrays Operating in Long Pulse Mode
}

\author{
Farzin Amzajerdian, Byron L. Meadows, Bruce W. Barnes, George E. Lockard, Upendra N. Singh, \\ Michael J. Kavaya \\ NASA Langley Research Center \\ MS 468, Hampton, Virginia 23681 \\ (757)864-1533 \\ f.amzajerdian@nasa.gov
}

Nathaniel R. Baker

Lockheed Martin Engineering and Science Company

Hampton, Virginia

\begin{abstract}
Reliability and lifetime of quasi-CW laser diode arrays are greatly influenced by their thermal characteristics. This paper examines the thermal properties of laser diode arrays operating in long pulse duration regime.

OCIS Codes: (140.2010) Diode laser arrays; (280.3640) Lidar
\end{abstract}

Most moderate to high power solid state lasers require high-power quasi-CW laser diode arrays (LDAs) as their pump source. Compared with their low-power CW counterparts, these LDAs suffer from shorter lifetimes and are more susceptible to premature failure. This is mainly due to the excessive localized heating and substantial pulse-to-pulse thermal cycling of the laser active regions. The thermally-induced stresses are even more dramatic when the required pump pulsewidth is increased from 200 microseconds, applied to neodymium-based lasers, to about 1 millisecond required for thulium and holmium lasers. Presently, lifetime testing of a number of LDAs from different suppliers is underway to assess the impact of long pulsewidth operation on the laser lifetime and reliability. The measurements to date indicate a lifetime of the order of only 200-300 million shots which is more than an order of magnitude shorter than reported lifetime for similar type of arrays operated at 200 microseconds pulsewidth. In addition to shorter lifetime, the arrays experience a high rate of catastrophic failure when operated in long pulse regime.

Though high power quasi-CW laser diodes arrays are complex electro-optical components and thus do not follow well defined or known predictable models, their lifetime, as electronic devices, may be estimated by an Arrhenius relationship written as:

$$
\text { Lifetime }(\tau) \propto\left(\mathrm{T}_{\mathrm{a}}-\mathrm{T}_{\mathrm{b}}\right)^{-\mathrm{N}} \operatorname{Exp}\left(\mathrm{E}_{\mathrm{a}} / \mathrm{kT}_{\mathrm{a}}\right)
$$

Where lifetime $(\tau)$ is expressed as a function of junction temperatures $T_{a}$ and $T_{b}$ measured immediately after and before the generated pulse, the activation energy $\left(E_{a}\right)$ and Boltzmann's constant $(k)$. The leading term accounts for the thermal cycling fatigue due to mismatch of thermal expansion coefficients of different package materials and various layers of the laser bar. The power $\mathrm{N}$ in expression above can have a value between 2 and 5 based on the Manson-Coffin law for thermal fatigue.

Several experimental setups have been developed to investigate the thermal characteristics of high power LDAs in order to evaluate various package designs, define the best operating parameters, and to guide the technology advancement, leading to highly reliable and very long lifetime LDAs. The thermal characterization of LDAs includes the thermal imaging of the facets, spectral shift and broadening measurements, and a forward voltage measurement technique we refer to as "Forward Voltage-Short Pulse” (FV-SP) technique. The FV-SP technique is devised based on a conventional measurement used for electrical diodes; light-emitting diodes, and continuous-wave laser diodes. This paper discusses the experimental methodology and the resulting data revealing the thermal characteristics of different LDAs and providing the bases for a meaningful trade analysis leading to maximum attainable lifetime and reliability. Such a trade analysis can enable the LDA specifications of the package type, number of bars per package and their pitch, operational temperature, and operating parameters such repetition rate, pulsewidth, and applied current. 